\title{
Numerical simulation of non-Archie electrophysi- cal property of saturated rock with lattice Boltzmann method
}

\author{
Yue Wenzheng ${ }^{1,2,3 *}$, Tao Guo ${ }^{1,2,3}$, Liu Dongming ${ }^{4}$ and Yang Wendu ${ }^{5}$ \\ ${ }^{1}$ State Key Laboratory of Petroleum Resource and Prospecting, China University of Petroleum, Beijing 102249, China \\ ${ }^{2}$ Key Laboratory of Earth Prospecting and Information Technology, China University of Petroleum, Beijing 102249, China \\ ${ }^{3}$ Research Centre of Well Logging, CNPC Key Laboratory of Well Logging, China University of Petroleum, Beijing \\ 102249, China \\ ${ }^{4}$ CNPC Logging Changqing Business Division, Xi’an, Shaanxi 710201, China \\ ${ }^{5}$ Sichuan Petroleum Administration Logging Company Ltd., CNPC, Chongqing 401147, China
}

\begin{abstract}
The electrophysical property of saturated rocks is very important for reservoir identification and evaluation. In this paper, the lattice Boltzmann method (LBM) was used to study the electrophysical property of rock saturated with fluid because of its advantages over conventional numerical approaches in handling complex pore geometry and boundary conditions. The digital core model was constructed through the accumulation of matrix grains based on their radius distribution obtained by the measurements of core samples. The flow of electrical current through the core model saturated with oil and water was simulated on the mesoscopic scale to reveal the non-Archie relationship between resistivity index and water saturation $\left(I-S_{\mathrm{w}}\right)$. The results from LBM simulation and laboratory measurements demonstrated that the $I-S_{\mathrm{w}}$ relation in the range of low water saturation was generally not a straight line in the log-log coordinates as described by the Archie equation. We thus developed a new equation based on numerical simulation and physical experiments. This new equation was used to fit the data from laboratory core measurements and previously published data. Determination of fluid saturation and reservoir evaluation could be significantly improved by using the new equation.
\end{abstract}

Key words: Non-Archie relation, digital core model, lattice Boltzmann method, numerical simulation, rock physical experiment

\section{Introduction}

The $I-S_{\mathrm{w}}$ relation of a formation is important for reservoir evaluation with well logging data. Archie (1942) first formulated the $I-S_{\mathrm{w}}$ relation based on a large number of core experiments on the sandstones of the Gulf of Mexico.

$$
I=b S_{\mathrm{w}}^{-n}
$$

where $I$ is the resistivity index, $S_{\mathrm{w}}$ is the water saturation of rock, $n$ is the saturation exponent and $b$ is the Archie parameter.

In the Archie equation, the $I-S_{\mathrm{w}}$ relation is linear in the $\log$ - $\log$ coordinates. In the past decades, the non-Archie phenomenon of reservoir rocks, i.e., the $I-S_{\mathrm{w}}$ relation is not linear in the log-log coordinates as shown in Fig. 1, has been increasingly observed and reported by log analysts and petroleum engineers (Diederix, 1982; Li, 1989; Worthington, 2000; 2006; Man and Jing, 2001; 2002; Li et al, 2008).

*Corresponding author. email: yuejack1@sina.com Received July 13, 2008

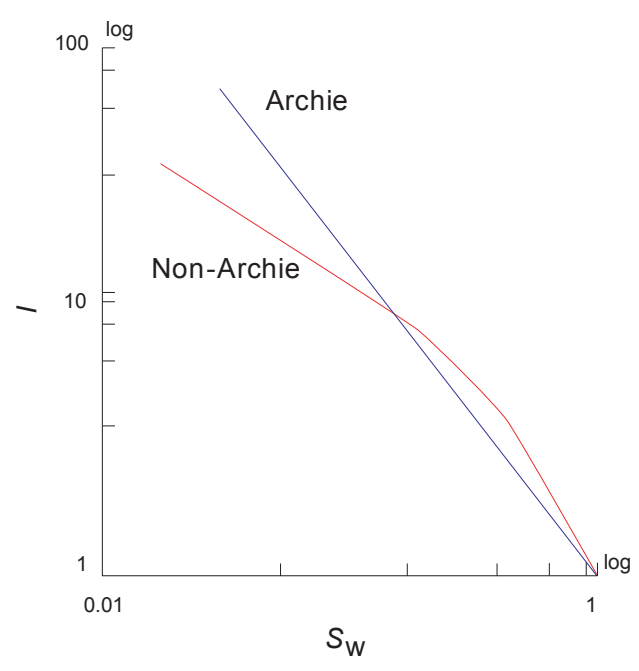

Fig. 1 Sketch of non-Archie $I-S_{\mathrm{w}}$ relation

These researchers have studied this so-called 'non-Archie phenomenon' of porous rocks extensively and have found that the non-Archie phenomenon generally becomes more evident 
as the water saturation further decreases. However, all these results also have shown that it is not possible to quantify the microscopic factors that influence the $I-S_{\mathrm{w}}$ relation, because the microscopic pore structure, the flow of fluid and electrical current in porous media cannot be directly observed and controlled in laboratory measurements. Consequently, many researchers have tried to simulate the phenomenon numerically on the pore scale (Tao, 1992; Man and Jing, 2001; 2002; Ding et al, 2004).

In the last decade, the lattice gas automata (LGA) method and the later lattice Boltzmann method (LBM) have developed rapidly in numerical simulation of fluid flow in porous media (Yan et al, 2006; Kutay et al, 2006). Yue et al (2005) studied the effects of matrix pore geometry, porefilling connate water and clay content on Archie equation with 2-D LGA method. In this paper, we further developed a numerical model based on LBM to investigate the electrical transport property of porous media saturated with fluid. Besides, we demonstrated the non-Archie phenomenon and compared the results of pore-scale numerical simulation with those of laboratory measurements. We also presented a more general $I-S_{\mathrm{w}}$ relation equation for reservoir porous rocks and used it to fit practical oilfield data.

\section{Construction of digital core model}

Most core samples used in laboratory studies are made of round grains from artificial materials, such as glass and aluminium. With the development of computer technology, numerical algorithms of uniform distribution of grain sizes and accumulation of random-sized grains to construct the core models have been developed and increasingly applied to producing virtual reality models of porous media to provide an insight into the physical properties of reservoir rocks. We describe an algorithm here to construct the digital core models with the sizes of matrix grains controlled by the porosity and the grain size distribution of core samples from laboratory measurements. The advantages of our method over conventional methods are that the distribution of grain sizes in digital core models can be identical to that in the real core samples. In this paper, the porous rock model used in the simulation is constructed through random accumulation of grains to form a pile of matrix grains with the algorithm.

In order to establish the porous rock model, the porosity and distribution of grain sizes of real core samples should be measured first. In Fig. 2, the vertical axis is the distribution of grain sizes obtained by laboratory measurements on core samples and the horizontal axis is the radius of grain with one unit increment of $0.015 \mathrm{~mm}$. Based on this distribution, we can construct the digital core model through accumulating the grains and compacting them into the limited space to satisfy the porosity for investigation of electrical property with LBM simulation. During the compaction, the grains may be moved from the original positions to new sites for reaching an equilibrium of contacted grains under pressure.

In Fig. 3, the map is the enlargement of a small portion of the constructed porous medium. To investigate the electrical property, the porous medium should be filled with fluid (oil and water in this case) to form the saturated porous medium model for revealing the effect of water saturation on the resistivity.

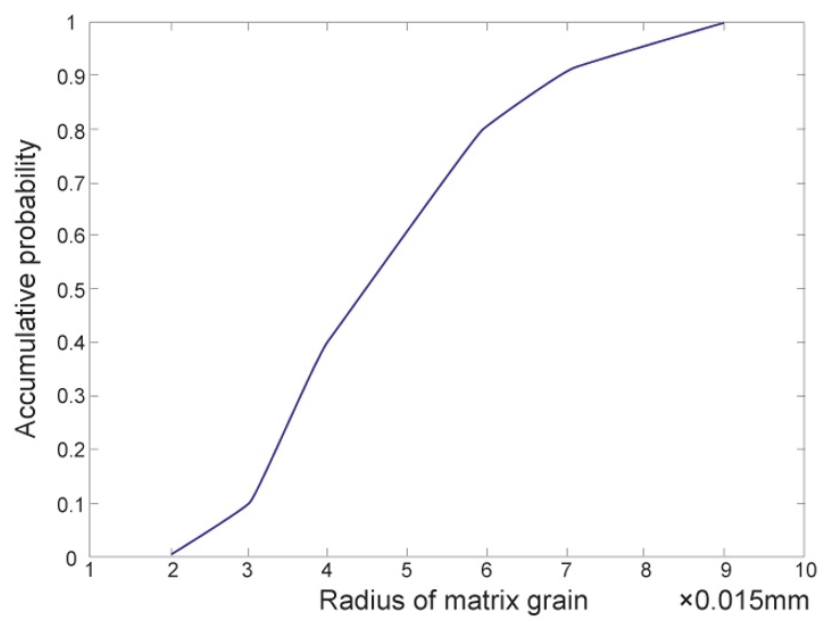

Fig. 2 Cumulative distribution of grain size of core samples

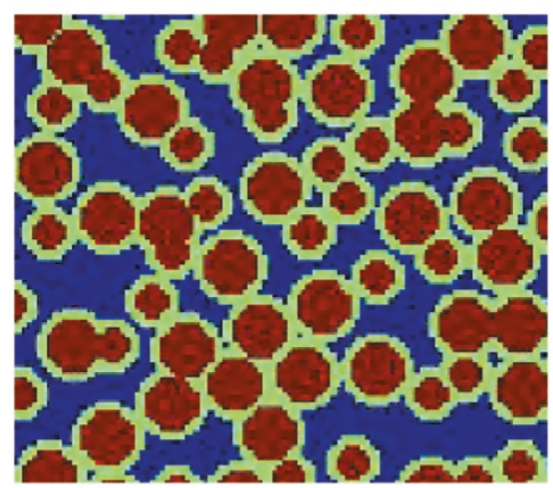

Fig. 3 Constructed porous medium model using our algorithm

For the simulation of resistivity with LBM, the saturated porous medium consists of three phases: matrix, water and oil. In this research, porosity is defined as a ratio of the nodes occupied by fluid to total nodes; water saturation is defined as a ratio of the nodes occupied by water to the nodes occupied by pore space.

In general, the complexity of the grain clusters can reflect the complexity of the pore space of real core samples because the model formed by accumulation of random distribution of grain sizes is identical to core samples in porosity and grain size distribution.

\section{Lattice Boltzmann method}

The LBM has been proved to be a powerful and flexible approach to mesoscopic numerical simulation, especially in monophase and multiphase fluid flow in porous medium, particle transfer, and heat convection (Cheng and Suo, 2003; Hazi, 2003; Ma et al, 2003; Haydock, 2005; Yan et al, 2006; Kutay et al, 2006; 2007; Boyd et al, 2006).

The LBM has developed to a series of discrete space 
models, including the two-dimensional model with multispeed since 1988 (Yan et al, 2006; Kutay et al, 2006). In the two-dimensional model (D2Q9), as shown in Fig. 4, the square lattice with nine velocity vectors is used in the discrete space of the computational domain and each node has eight neighbouring lattice nodes. At each time step, a particle at a node described by the distribution function of particle density moves to a neighbouring node, meanwhile, some particles move to this node together and undergo a collision at the next time step to form a new distribution of particles.

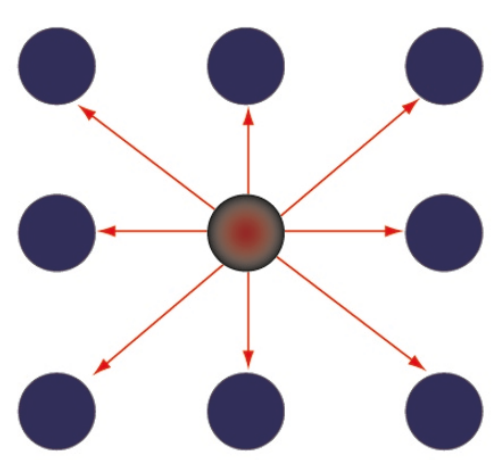

Fig. 4 Basic structure of D2Q9 model

The time evolution of LBM equation can be written as (Kutay et al, 2006)

$$
\begin{aligned}
& f_{i}\left(x+e_{i}, t+1\right)-f_{i}(x, t)=-\frac{1}{\tau}\left(f_{i}(x, t)-f_{i}^{e q}(x, t)\right) \\
& i=0,1,2,3, \ldots, 8
\end{aligned}
$$

It was proved that the Navier-Stokes equation (NSE) can be derived from the time evolution of LBM on the mesoscopic scale using multi-scale and Champman-Enskog expansion technology with definition of density and momentum as the function of particle distribution and unit velocity vector, respectively.

$$
\rho(x, t)=\sum_{i=0}^{b} f_{i}(x, t), \quad \rho u(x, t)=\sum_{i=0}^{b} f_{i}(x, t) e_{i}
$$

The numerical simulation involved in this study is modeling the flow of Newtonian fluid in a two-dimensional channel with rigid boundaries at the top and bottom. For stationary flow driven by a constant horizontal pressure gradient $\nabla P$, if the velocity is sufficiently low to neglect the inertia terms, the NS equation can be simplified to Darcy's law, i.e.,

$$
Q=K \nabla P / \mu
$$

where $Q$ is the volume flow rate in unit of $\mathrm{m} / \mathrm{s}, K$ is the permeability of the medium in unit of $\mathrm{m}^{2}$, and $\mu$ is the dynamic viscosity of the fluid.

On the other hand, in a continuous medium, Ohm's law relates the current density $j$ to electric field intensity $E$ by

$$
j=\sigma E=\sigma \nabla \phi
$$

where $\sigma$ is the electric conductivity, $\phi$ is the electric potential. The analogy between Darcy's law and Ohm's law is evident if electrons replace the fluid and the electric field replaces the pressure gradient. Since $K$ is constant for a Poiseuille flow, it appears that Eqs. (3) and (4) are equivalent if the resistivity corresponds to the effective viscosity of the fluid.

The physical model involved in this paper is presented in Fig. 5 with the rigid boundaries at the top and bottom corresponding to the insulated boundaries in the simulation of resistivity. The insulated condition is obtained by the boundary condition of half way rebound of particles with the collision happening between the boundary wall nodes and the fluid nodes of first layer through simply reversing the particle velocity $(u, v)$ to $(-u,-v)$. Periodic boundary conditions in the horizontal direction are approximated to an infinitely long current channel (Chen and Doolen, 1998).

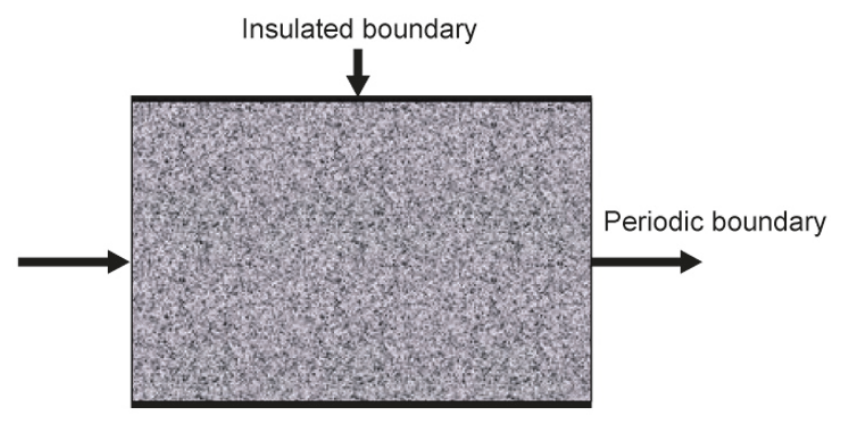

Fig. 5 Sketch of boundaries of porous medium

\section{Results and discussions}

The size of the porous medium model used in the simulation was $250 \times 200$ nodes. The constant force coefficient for electric field intensity was 0.0005 and the initial average density distribution of the particles for the LBM model was 1.0. Simulation convergence was assumed when the difference in velocity between two time steps was less than $10^{-6}$. Simulations were implemented for the models saturated with mixture of oil and water with different water saturations. In Fig. $6, I-S_{\mathrm{w}}$ relation can be formulated by the Archie equation and the saturation exponent $n$ is independent of $S_{\mathrm{w}}$ when $S_{\mathrm{w}}$ is more than 0.15 for a model with fixed porosity of 0.2. The non-Archie phenomenon will appear if water saturation is lower than 0.15 , and then the $I-S_{\mathrm{w}}$ relation deviates gradually to the water saturation axis non-linearly with decreasing water saturation. The results of rock physical experiments by Jing et al (1993) share the same conclusion with our numerical simulation for $I-S_{\mathrm{w}}$ relation. The same results were obtained from the $\mathrm{LBM}$ simulation of the constructed porous medium model with different porosities.

The simulated results, as shown in Fig. 6, clearly demonstrated that the relation of $I-S_{w}$ was affected by the distribution of fluid filling in pore space. When the water saturation was greater than the oil saturation, the water was the continuous conductive phase. The clusters of oil, formed with decreasing water saturation, increased the tortuosity of current path rapidly so that the resistivity index increased in 
an inverse power function of water saturation as described by the Archie equation. However, the change rate of tortuosity of the current path started to decrease gradually when the clusters of oil expanded to connect each other to form the continuous phase in the pore space after the water saturation was lower than 0.15 . Therefore, the nonlinear phenomenon of the $I-S_{\mathrm{w}}$ relation in the log-log coordinates might be caused by the non-constant change rate of the current path tortuosity due to the fluid distribution. It is clear that the fluid distribution is an important factor leading to the non-Archie phenomenon.

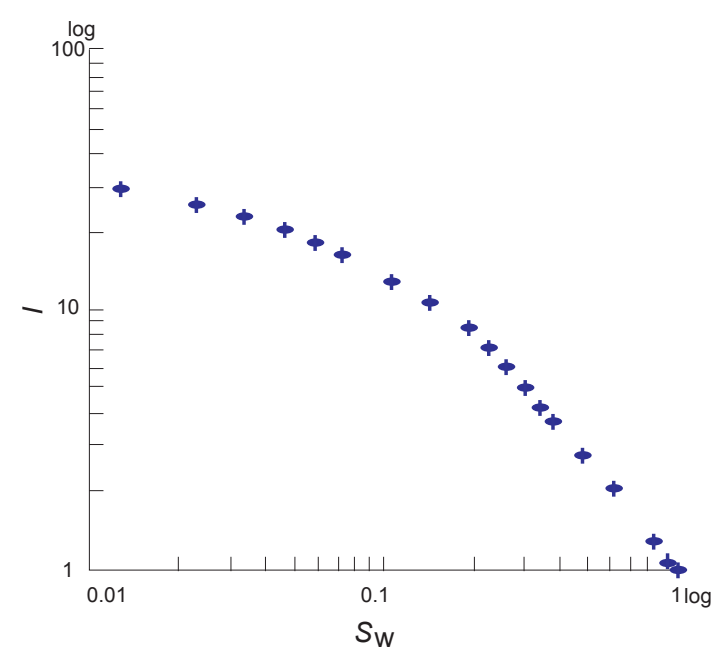

Fig. $6 I-S_{\mathrm{w}}$ relation obtained by LBM

By comparing the simulated results above with the physical experiments on core samples we did before, we can see that the $I-S_{\mathrm{w}}$ relation in the log-log coordinates is a curve, i.e., with a non-constant exponent $n$ changing with water saturation, instead of a straight line as described by the Archie equation. The Archie equation actually gives an approximate $I-S_{\mathrm{w}}$ relation in the range of high water saturation.

Having realized the fact that exponent $n$ was a function of water saturation, we thus developed a new equation of the non-Archie $I-S_{\mathrm{w}}$ relation for calculating fluid saturation and reservoir evaluation. This more general equation, based on the results of LBM simulation of the electrical property of a porous medium saturated with fluid, can be applied to calculating formation fluid saturation for the reservoir rocks of both Archie and non-Archie types. This new equation is essentially different from the Archie equation in that the saturation exponent $n$ is no longer a constant but a function of water saturation as below:

$$
I=S_{\mathrm{w}}^{-Y S_{\mathrm{w}}^{U}}
$$

where $Y$ and $U$ are two constants related to pore structure and porosity.

To demonstrate the applicability of this new equation, especially for the non-Archie rocks, we used both equations to fit the data obtained by laboratory measurements to observe the differences between them.

To show the data more clearly without losing generality, we plotted here only one of the eight data groups in Fig. 7.
Actually, all the eight data groups are similar. In this figure, the solid line is the result calculated by our new equation while the dashed line is the result of Archie equation. The diamonds are the data from laboratory measurements. It is clear that this new equation fits the measured data better than the Archie equation and therefore represents a more precise conductivity model and a more actual mechanism of current flow in fluid saturated porous medium. It can lead to a more precise evaluation of oil-bearing reservoirs with a high resistivity index.

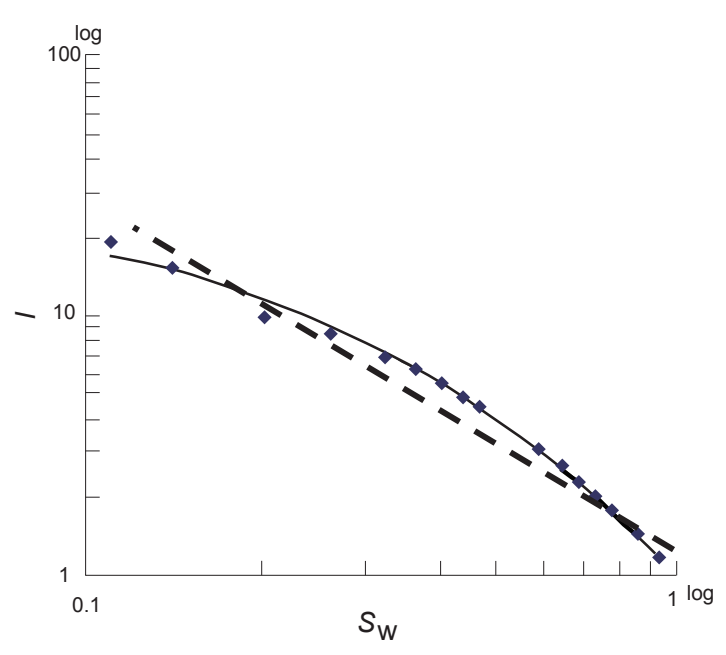

Fig. 7 Fitting results of the measured data using the new equation and Archie equation respectively

\section{Conclusions}

1) An algorithm was proposed to construct the digital core models with the sizes of matrix grains controlled by the porosity and the grain size distribution of core samples from laboratory measurements. The advantages of our method lie in that the distribution of grain sizes of digital core models can be identical to that of the real core samples.

2) The lattice Boltzmann method was used to study the electrophysical property of rock model saturated with fluid. The simulated results clearly demonstrated that the relation of $I-S_{\mathrm{w}}$ was affected by the distribution of fluid filling in pore space. The non-Archie phenomenon would appear if water saturation was lower than 0.15 , and the $I-S_{\mathrm{w}}$ relation deviated gradually to the water saturation axis non-linearly with decreasing water saturation.

3) Based on the results, we developed a new equation for the non-Archie $I-S_{\mathrm{w}}$ relation for calculating fluid saturation and reservoir evaluation. This new equation was used to fit the data from laboratory core measurements and previously published data. Determination of fluid saturation and reservoir evaluation could be significantly improved by using the new equation.

\section{Acknowledgements}

This research was sponsored by the project No. 50404001 from the National Natural Science Foundation of China, the National Key Fundamental Research \& Development Project 
(Grant No. 2007CB209601), the China National Petroleum Corporation Fundamental Research Program (Grant No. 06A30102) and the China Postdoctoral Science Foundation (Project No. 2004035350).

\section{References}

Archie G E. The electrical resistivity log as an aid in determining some reservoir characteristics. Trans. AIME. 1942. 146: 54-61

Boyd J, Buick J and Green S. A second-order accurate lattice Boltzmann non-Newtonian flow model. Journal of Physics A: Mathematical and General. 2006. 39: 14241-14247

Cheng Y and Suo L S. Lattice Boltzmann scheme to simulate twodimensional fluid transient. Journal of Hydrodynamics B. 2003. 2: 19-23

Chen S Y and Doolen G D. Lattice Boltzmann method for fluid flow. Annual Review of Fluid Mechanics. 1998. 30: 329-364

Diederix K M. Anomalous relationships between resistivity index and water saturations in the rotliegend sandstone. Transaction of SPWLA 23rd Annual Logging Symposium. 1982. Paper X

Ding H, Shu C, Yeo K S, et al. Simulation of incompressible viscous flows past a circular cylinder by hybrid FD scheme and meshless least square-based finite difference method. Computer Methods in Applied Mechanics and Engineering. 2004. 193(9-11): 727-744

Haydock D. Lattice Boltzmann simulations of the time-averaged forces on a cylinder in a sound field. Journal of Physics A: Mathematical and General. 2005. 38: 3265-3277

Hazi G. Accuracy of the lattice Boltzmann method based on analytical solutions. Physical Review E. 2003. 67(5): 056705.1-056705.5

Jing X D, Gillespie A and Trewin B M. Resistivity index from nonequilibrium measurements using detailed in-situ saturation monitoring. Society of Petroleum Engineers Paper. Offshore European Conference, Aberdeen. 1993. 456-464

Kutay M E, Aydilek A H and Masad E. Laboratory validation of lattice Boltzmann method for modeling pore-scale flow in granular materials. Computers and Geotechnics. 2006. 33(8): 381-395
Kutay M E, Aydilek A H, Masad E, et al. Computational and experimental evaluation of hydraulic conductivity anisotropy in hotmix asphalt. International Journal of Pavement Engineering. 2007. 8(1): $29-43$

Li N. General forms of the resistivity-porosity and resistivity-oil/gas saturation relations, as well as the determination of their optimum approximation function types. Chinese Journal of Geophysics. 1989. 32(5): 580-591 (in Chinese)

$\mathrm{Li} \mathrm{X} \mathrm{D,} \mathrm{Yu} \mathrm{J} \mathrm{and} \mathrm{Li} \mathrm{M.} \mathrm{Theoretic} \mathrm{research} \mathrm{of} \mathrm{reservoir} \mathrm{rock} \mathrm{with} \mathrm{non-}$ Archie characteristics. Oil-Gasfield Surface Engineering. 2008. 27(1): 32-33 (in Chinese)

Ma B, Lei S Y, Hao J Z, et al. Simulation of fluid flow in micro-channel by lattice Boltzmann method. Journal of Guangxi Normal University (Natural Science Edition). 2003. 21(2): 20-24 (in Chinese)

Man H N and Jing X D. Network modelling of strong and intermediate wettability on electrical resistivity and capillary pressure. Advances in Water Resources. 2001. 24(3-4): 345-363

Man H N and Jing X D. Network modelling of mixed-wettability on electrical resistivity, capillary pressure and wettability indices. Journal of Petroleum Science and Engineering. 2002. 33(1-3): 101-122

Tao G. Elastic and transport properties of some sandstones (PhD thesis). London: Imperial College of Science, Technology \& Medicine, University of London. 1992

Worthington P F. Recognition and evaluation of low-resistivity pay. Petroleum Geoscience. 2000. 6(1): 77-92

Worthington P F. Quality assurance of the evaluation of hydrocarbon saturation from resistivity data. SPE Annual Technical Conference and Exhibition, Texas. 2006. Paper 103075

Yan G W, Dong Y F and Liu Y H. An implicit Lagrangian lattice Boltzmann method for the compressible flows. International Journal for Numerical Methods in Fluids. 2006. 51(12): 1407-1418

Yue W Z, Tao G and Zhu K Q. Simulation of electrical transport properties in oil-water saturated porous media with 2-D lattice gas automata. Chinese Journal of Geophysics. 2005. 48(1): 189-195 (in Chinese)

(Edited by Hao Jie) 